\title{
Voltage sag source location in distribution networks
}

\author{
E. Belenguer ${ }^{1}$, C. Reineri ${ }^{2}$, N. Aparicio ${ }^{1}$ and R. Mampel ${ }^{1}$ \\ ${ }^{1}$ Department of Technology \\ Universitat Jaume I \\ Campus de Riu Sec, 12071 Castellón (Spain) \\ phone:+34 964 728176, fax:+34 964 7281076, e-mail: efbeleng@tec.uji.es \\ ${ }^{2}$ Department of Electrical Engineering \\ Universidad Nacional de Río Cuarto \\ Ruta 36 km 601, 5800 Río Cuarto (Argentina) \\ phone:+54 358 676246, fax:+54 358 676246, e-mail: creineri@ing.unrc.edu.ar
}

\begin{abstract}
This paper describes a method developed to determine, in a distribution system, the relative location of a voltage sag source according to the geographical position of a recording device. The method is based on the analysis of the change in the instantaneous power (calculated from the line voltage and current waveforms recorded by the device) during the transient state.

For the development of the method, a low-voltage electrical model of a simplified distribution network has been used in order to simulate, in a controlled way, the main types of voltage sag sources. The results obtained with this model have been checked up by applying the method procedure to a number of real system voltage and current records.
\end{abstract}

\section{Key words}

Power quality, voltage sags, disturbance origin location.

\section{Introduction}

One of the main problems related to power quality in distribution networks is the existence of voltage sags. In developed countries, it is known that from $75 \%$ up to 95 $\%$ of the industrial sector claims to the electric distribution companies are related to problems originated by this disturbance type [1], [2]. These problems arise from the fact that many electrical loads are not designed to maintain their normal use behaviour during a voltage sag. Electrical motors and drives, frequency converters, programmable logic controllers and other electronic loads are sensitive equipment, i.e., "they are not able to work properly during the presence of the perturbation or cannot continue working without the intervention of an operator". Related to this, the standard EN 61000-3 defines many electromagnetic compatibility requirements that electrical equipment must meet. On the other side, the EN 50160 describes the voltage characteristics of a public distribution system supply. The real situation is, however, that equipment made to meet the first standard may be affected by an electrical supply that accomplishes the second one.

The concept of sensitive equipment may be extended to an industrial production process (sensitive process) when it may be affected (interrupted) by the presence of electrical disturbances. This is the case of the ceramic industry in the province of Castellón (Spain) which production processes have a high degree of automation and are very often affected by the presence of voltage sags. It is important to remind that a single voltage sag may be followed by a costly process downtime, lost production and, even, damage of equipment.

In the frame of a regulated energy market, it would be quite useful to define quality of supply indicators and establish their associated electromagnetic compatibility levels so that financial penalties may be imposed to the responsible party for generating a disturbance resulting in customer downtime. This has been done for harmonic pollution in some countries where models and methods have been developed to, first, find the origin of the disturbance and, next, apply a penalization to the responsible party. Similarly, power quality indicators may be needed, in the near future, for the case of voltage sags and, accordingly, techniques to locate the disturbance source will have to be developed.

In this study, a method has been developed to determine, once a voltage sag has spread into the distribution network, the relative location of the disturbance source according to the position of a recording device. If several devices are distributed throughout the network, it is possible to determine more accurately the source location.

The method is based on the analysis of the three-phase instantaneous power calculated from the voltage and current waveforms measured by a recording device. To be exact, the analysis is made on the change in the 
instantaneous power during the transient state produced by the voltage sag.

A similar method that can be applied, according to the authors, to any type of perturbation is explained in [3]. However, the limited number of voltage sag cases considered in this paper and the complexity of a real network makes it advisable to extend the analysis to a number of different voltage sag causes.

\section{Principles of the location method}

It is well known that the presence of a voltage sag in the distribution network may be produced by a number of different causes which origin can be located at the different voltage levels of the power system. Although, voltage sags are mainly caused by faults (short-circuits), there are other sources that have to be considered and studied. Fig. 1 shows the main voltage sag sources according to their location in the power system.

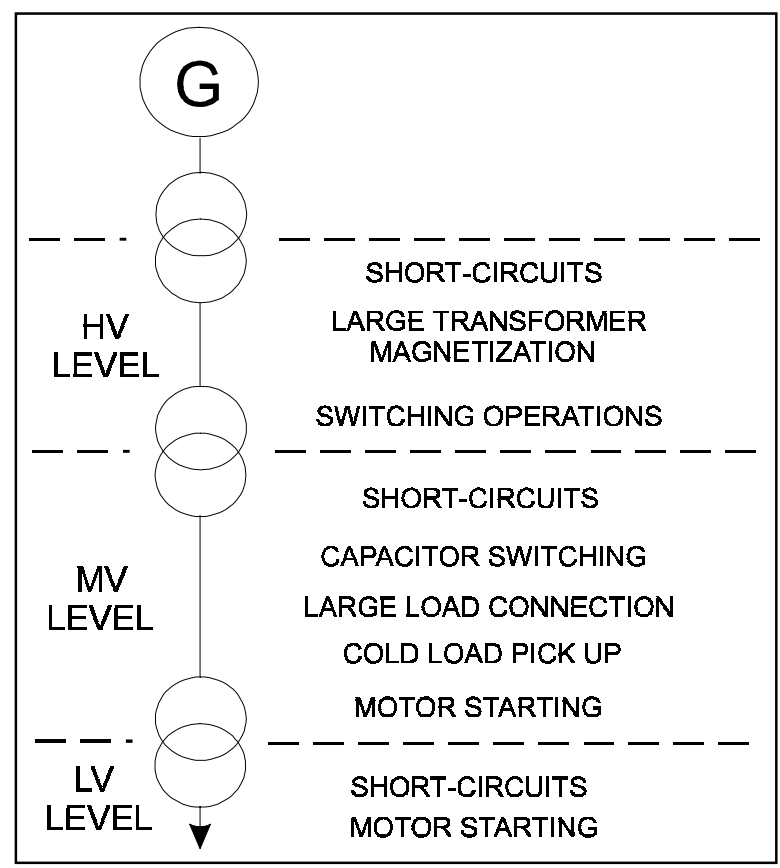

Fig. 1. Possible voltage sag sources according to the system level

Our main objective is to find out on which side of the recording device the voltage sag originates. In this respect, the first step should be to define a reference direction for the recording equipment. We define the positive direction as the energy flow direction from the substation to the load. Fig. 2 shows a simplified distribution network where several recording devices have been placed. For each device a reference direction has been established.

The method presented in [3] is based on the idea that a transient disturbance source may be considered as an energy sink. Consequently, if the source is located in front of the recording device (positive direction), more energy will flow in that direction. Inversely, if the source is located behind the device (negative direction), there will be a decrease in the energy supplied to the load connected in front of the device. Although this idea may be applied to some disturbance sources, such as capacitor switching or resistive short-circuits, it is quite obvious that cannot explain the behaviour of other disturbance sources.

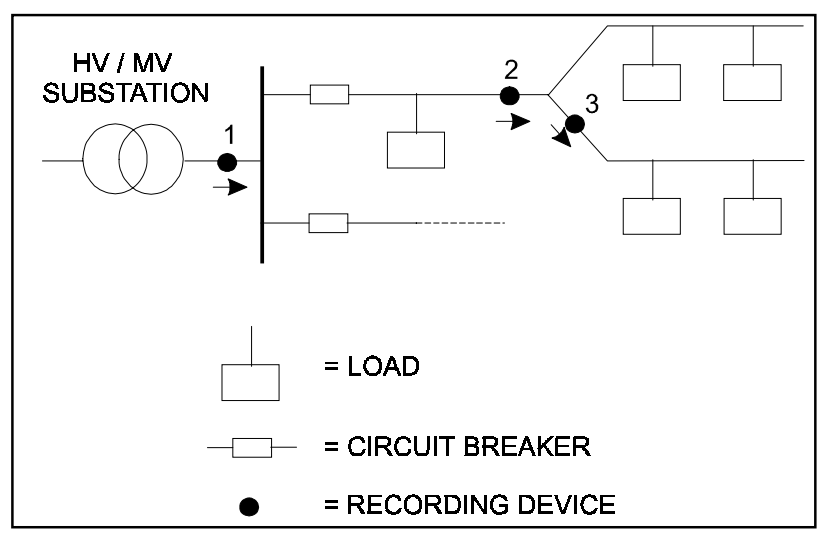

Fig. 2. Simplified distribution network

For the case of voltage sags, we have to consider that if the source is located behind the recording device, the downstream load will be affected by the sudden reduction of the voltage and there will be, in the general case, a decrease in the power or energy supplied to the load.

On the other hand, if the voltage sag source is located in the positive direction of the recording device (e.g. a large motor starting in that direction), the power decrease in the rest of the loads (due to the voltage sag) may be compensated by the extra energy supplied to the disturbance source during the transient period. Considering the characteristics of the usual electrical elements that make up a real electrical system, it is quite reasonable to say that the system will respond with an increase in the power consumed by the total load located in the positive direction.

Anyway, the number of different possible voltage sag sources together with the increase in the use of highly non linear loads with an unpredictable behaviour, lead us to test real systems and check up the previous statements.

\section{Testing and results}

The testing procedure has been carried out in a double direction. On one side, a low-voltage electric circuit equivalent to a simplified distribution network has been built with the objective of analysing the instantaneous power under controlled voltage sag disturbances. In addition to this, current and voltage records from a real distribution network have been used to calculate the trend of the instantaneous power from the disturbance starting point.

Both procedures and their results are shown below. 


\section{A. Equivalent low-voltage physical model}

Fig. 3 shows the low-voltage three-phase circuit used to evaluate the instantaneous power under voltage sag conditions caused by different sources. To model the presence of a voltage sag originated at the HV side of the substation, a three-phase programmable power supply has been used. For the analysis of other sources at the load side, such as motor starting and large load connection, the circuit has been directly supplied by the low-voltage network.

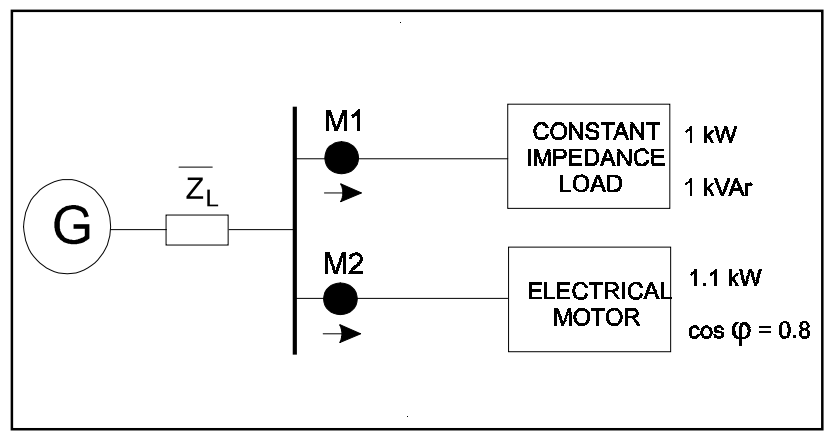

Fig. 3. Equivalent low-voltage physical model

Fig. 4 shows the phase voltages and line currents recorded by device M1 and the instantaneous power in the case of a single-phase voltage sag (case 1) produced by the programmable power supply (with this we simulate a voltage sag source located at the HV side of the network). As expected, there is a decrease in the mean value of the instantaneous power determined in M1 (from $367 \mathrm{~W}$ to $307 \mathrm{~W}$ as shown in the figure). It must be stressed that the initial peak of the instantaneous power is negative following the general energy decreasing trend.

The same behaviour is verified using the measurements recorded by device M2 as shown in Fig. 5, although the instantaneous power mean value reduction is not so pronounced. In both cases, these two characteristics: (i) a decrease in the power mean value and (ii) an initial negative power peak, give us the indication that the voltage sag source is located in the negative direction with respect to both recording devices.

Next, we consider the case of direct motor starting (case 2 ), producing large initial currents and, consequently, a voltage sag that can persist for several seconds. Fig. 6 shows the results for the device M1 indicating, as in the previous examples, that the disturbance source is in the negative direction (behind M1). Inversely, the positive initial peak of the instantaneous power for the device M2 (Fig. 7), points out a positive direction.

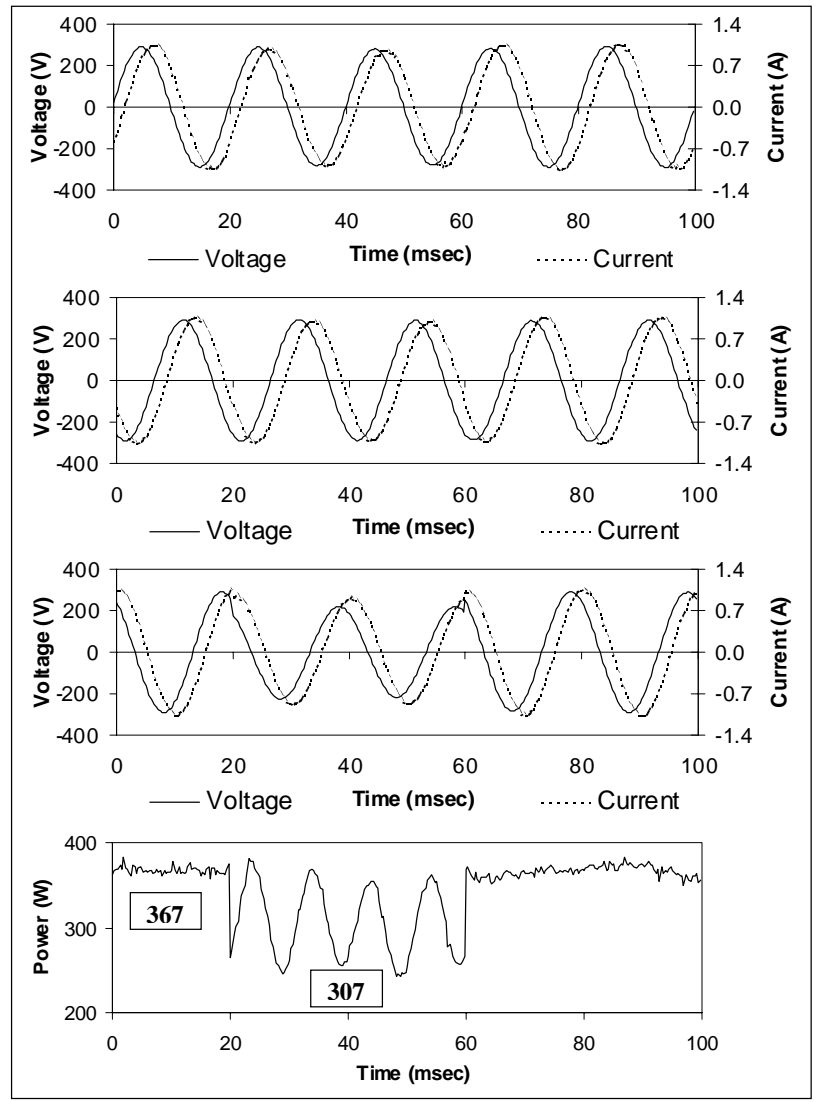

Fig. 4. Case 1 recorded event at M1

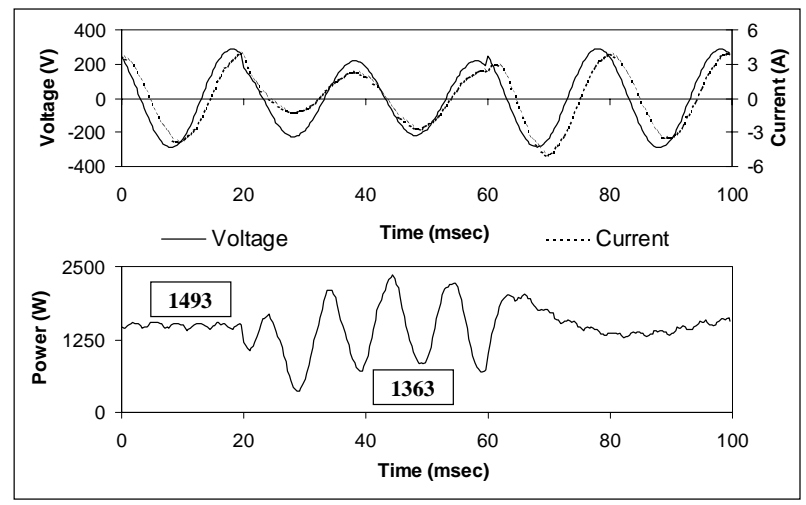

Fig. 5. Case 1 recorded event at M2

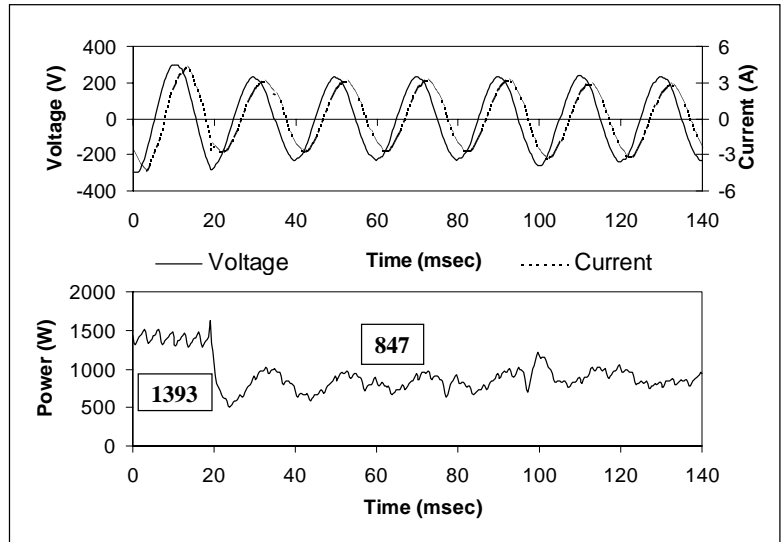

Fig. 6. Case 2 recorded event at M1 


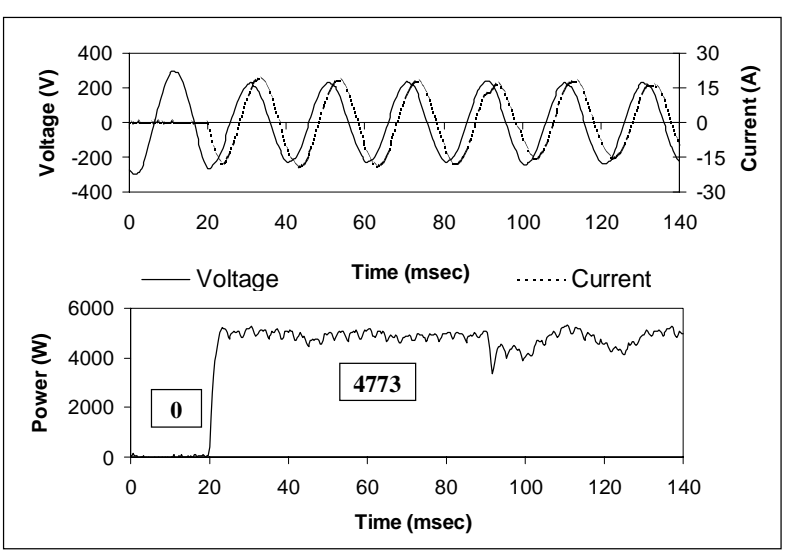

Fig. 7. Case 2 recorded event at M2

Finally, a third type of voltage sag source is simulated (case 3) through the connection of a large single-phase resistive load in parallel with the constant impedance load (a similar effect would be the occurrence of a singlephase resistive fault in the same connection point). Figures 8 and 9 show, respectively, that the source is located in front of M1 (positive direction) and behind M2 (negative direction).

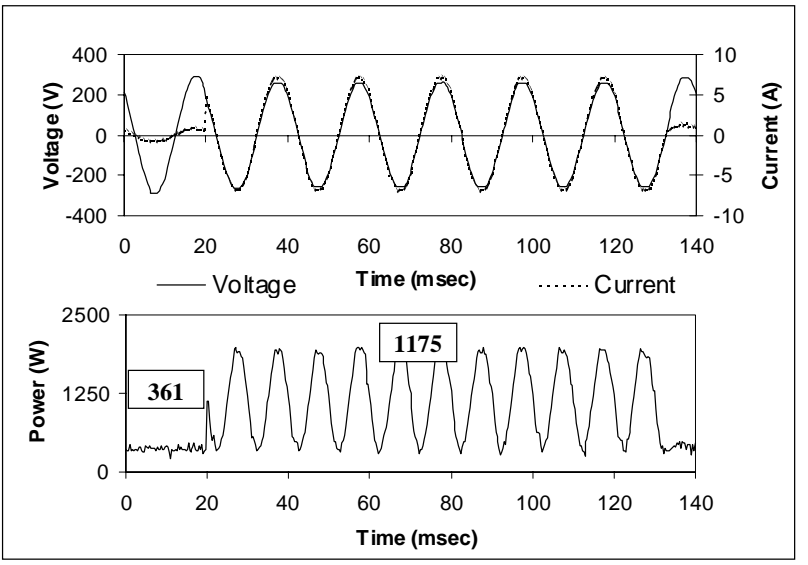

Fig. 8. Case 3 recorded event at M1

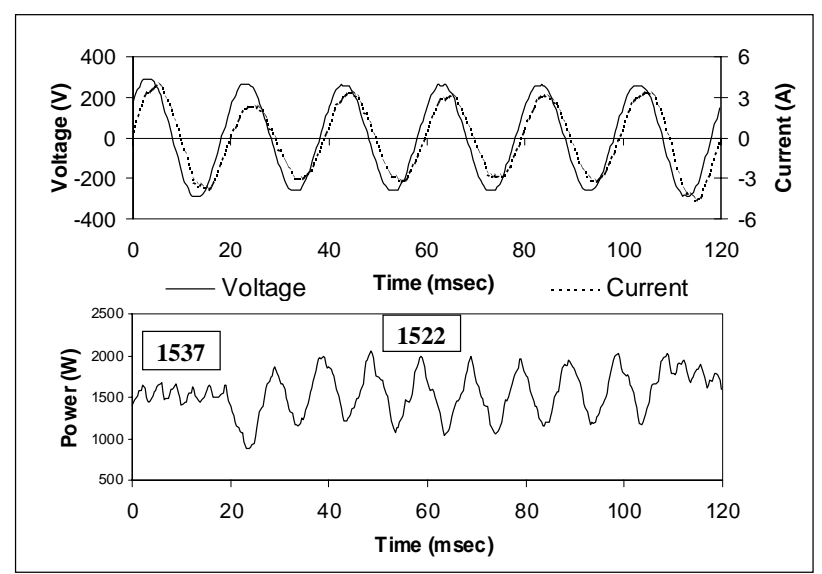

Fig. 9. Case 3 recorded event at M2

\section{B. Real records in a distribution system}

In order to completely verify the proposed method, it has been applied to voltage sag events recorded at a $33 \mathrm{kV}$ distribution system in Argentina.

Fig. 10 shows a recorded voltage sag and the corresponding instantaneous power. In this case, it was known that the voltage sag was produced by a fault at a feeder different to the one where the recording device was connected. Again, the polarity of the initial peak and the reduction of the mean value indicate the right location of the source, i.e. negative direction. Similar results with a large number of records, not shown in this paper, confirm the validity of the method.

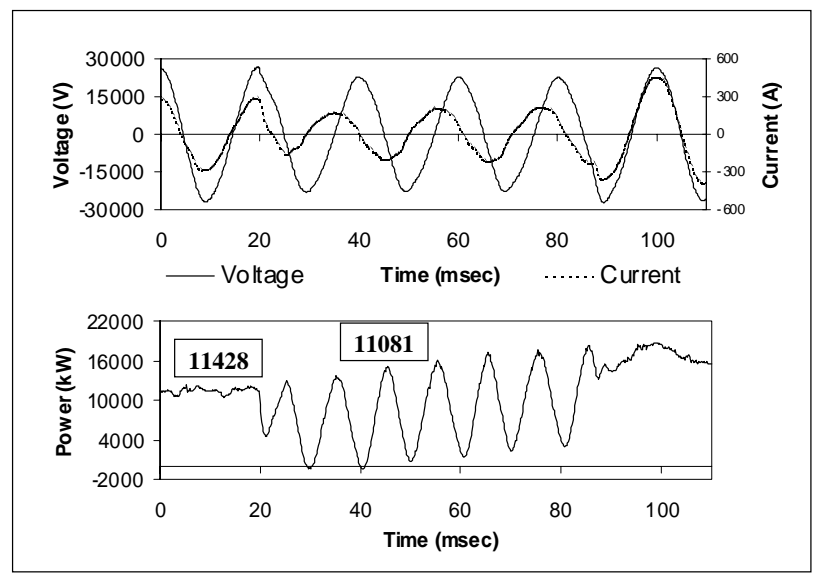

Fig. 10. Recorded event at a $33 \mathrm{kV}$ distribution system

The analysed records have shown us that the method can be applied not only to voltage sags but also to other disturbance types. As an example, it is shown in Fig. 11 the case of the connection of a transformer downstream from the position of the recorder. In this case there is an increase in the mean value of the instantaneous power and the polarity of the initial peak is positive, what indicates a positive direction for the source.

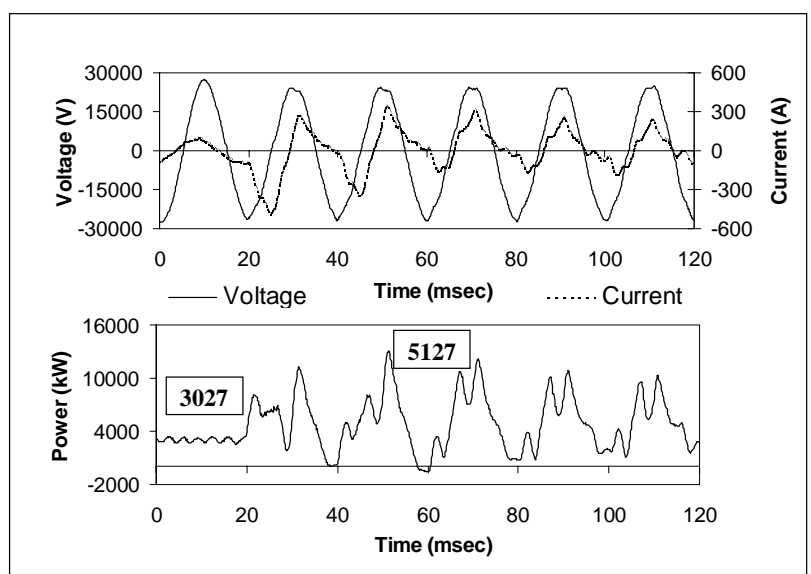

Fig. 11. Transformer connection at a $33 \mathrm{kV}$ distribution system 


\section{Method description}

The complete procedure to find out the location of a voltage sag source is described now.

- $\quad$ Line voltage and current waveforms, before and during the disturbance, are recorded at a specified point of the distribution network where a recording device is located. A reference direction is established for the device.

- The three-phase instantaneous power is determined using the voltage and current samples.

- The mean value of the power, before and during the disturbance is calculated.

- The polarity of the initial peak of the power is considered.

There is a simple rule to determine the voltage sag source location:

- If the polarity of the initial peak of the instantaneous power is negative or there is a reduction in the power mean value after the beginning of the event, then the voltage sag source is located in the recording device negative direction.

- Inversely, if the polarity of the initial peak of the instantaneous power is positive or there is an increase in the power mean value after the beginning of the event, then the voltage sag source is located in the recording device positive direction.

\section{Power quality monitoring system}

It is very understandable that the existence of just one recording device in a distribution network can give little information about the location of the voltage sag source. However, if the utility decides to implement a complete power quality monitoring system with a number of recorders, then the proposed method can be very useful to locate the responsible party for generating the disturbance.
If we consider again Fig. 2, we easily see that concurrent measurements from the three recording devices can help the power engineer to locate, in a much more accurate way, the origin of the disturbance. Taking into account the directions indicated for each recorder it is possible to determine, in this case, up to four possible areas for the source location.

\section{Conclusions}

A method for locating the origin of a voltage sag disturbance in a distribution network has been presented. It is based on the analysis of the instantaneous power calculated from voltage and current waveforms recorded at one or more points of the distribution system. The method implements the capability to determine whether the voltage sag originates in front of or behind the recording device.

The different results shown in the paper demonstrate that the method is applicable to the main voltage sag sources.

In addition to this, some results obtained from the different cases considered, show that the method can be applied to other disturbance types.

\section{Acknowledgement}

The authors are grateful for the financial support provided by the Universitat Jaume I Research Development Programme.

\section{References}

[1] M. Bollen, Understanding Power Quality Problems: Voltage Sags and Interruptions, IEEE Press in New York, ISBN: 0-7803-4713-7

[2] J. C. Gómez, M. M. Morcos, C. A. Reineri, N. G. Campetelli, "Behaviour of Induction Motors due to Voltage Sags and Short Interruptions", IEEE Trans. on Power Delivery, Vol. 7, No. 2, pp 434-440, April 2002.

[3] A. C. Parsons, W. Grady, J. C. Soward, “A Direction Finder for Power Quality Disturbances Based Upon Disturbance Power and Energy", IEEE Trans. on Power Delivery, Vol. 15, No. 3, pp 1081-1086, July 2000. 